Europhysics Letters

PREPRINT

\title{
Specific heat and disorder in the mixed state of non- magnetic borocarbides
}

\author{
D. $\operatorname{Lipp}^{1}\left(^{*}\right)$, M. Schneider ${ }^{1}$, A. Gladun ${ }^{1}$, \\ S.-L. Drechsler ${ }^{2}$, J. Freudenberger ${ }^{2}$, G. Fuchs ${ }^{2}$, K. Nenkov ${ }^{2}$, K.-H. MÜller ${ }^{2}$, \\ T. CiChoreK ${ }^{3}$ and P. Gegenwart ${ }^{3}$ \\ 1 Institut für Tieftemperaturphysik, Technische Universität Dresden, D-01062 Dresden, \\ Germany \\ 2 Institut für Festkörper- und Werkstofforschung, D-01171 Dresden, Postfach 270116, \\ Germany \\ 3 Max-Planck-Institut für Chemische Physik fester Stoffe Dresden, D-01187 Dresden, \\ Germany
}

PACS. 74.70.Dd - borocarbides.

PACS. 74.62.Dh - rare earth and transition metal substitution.

PACS. 74.25.Bt - specific heat.

\begin{abstract}
The temperature and magnetic field dependence of the specific heat $c_{p}(T, H)$ in the superconducting mixed state as well as the upper critical field $H_{c 2}(T)$ have been measured for polycrystalline $\mathrm{Y}_{x} \mathrm{Lu}_{1-x} \mathrm{Ni}_{2} \mathrm{~B}_{2} \mathrm{C}$ and $\mathrm{Y}\left(\mathrm{Ni}_{1-y} \mathrm{Pt}_{y}\right)_{2} \mathrm{~B}_{2} \mathrm{C}$ samples. The linear-in- $T$ electronic specific heat contribution $\gamma(H) \cdot T$ exhibits significant deviations from the usual linear-in- $H$ law for all $x$ and $y$ the transition metal site $(T)$ resulting in a disorder dependent negative curvature of $\gamma(H)$. The deviations from that linear behaviour of our unsubstituted samples are the largest reported so far for any superconductor. The $H_{c 2}(T)$ data point to the quasi-clean limit for $(\mathrm{Y}, \mathrm{Lu})$-substitutions and to a transition to the quasi-dirty limit for $(\mathrm{Ni}, \mathrm{Pt})$-substitutions. The $\gamma(H)$ dependence is discussed in the unitary $d$-wave as well as in the quasi-clean $s$-wave limits. From a consideration of $\gamma(H)$ data only, $d$-wave pairing cannot be ruled out.
\end{abstract}

INTRODUCTION. - The rare earth $(R)$ transition metal $(T)$ borocarbide family $(R \mathrm{C})_{n} T_{2} \mathrm{~B}_{2}(R=\mathrm{Y}, \mathrm{Lu}, \mathrm{Sc}$, Th, La; $T=\mathrm{Ni}, \mathrm{Pd}, \mathrm{Pt} ; n=1$ or 2$)$ contains superconductors with relatively high transition temperatures $T_{c}$ up to $23 \mathrm{~K}$ [1, 2]. The coexistence of superconductivity and magnetism for members of this family where $R$ are magnetic rare earth ions such as Dy, Ho, Er, ... , has stimulated numerous studies of their thermodynamic and transport properties in the superconducting as well as in the normal state. At first glance, most of those results support a classification of these materials as intermetallic phonon mediated superconductors with a moderately strong coupling strength. However, clean $R \mathrm{Ni}_{2} \mathrm{~B}_{2} \mathrm{C}$ samples exhibit also some features unexpected for ordinary $s$-wave superconductors. We emphasize the unusual shape and the strong disorder dependence of the upper critical field $H_{c 2}(T)$ and a

$\left(^{*}\right)$ E-mail: lipp@physik.phy.tu-dresden.de; Fax: +49-351-463-7060

(c) EDP Sciences 
nearly $T^{3}$ scaling of the electronic specific heat $c_{e s}(T)$ in the superconducting state compared with exponential behaviour for ordinary $s$-wave superconductors [3].

According to Nohara et al. [4] the isoelectronic $T$-substitution does affect strongly the field dependence of the linear-in- $T$ electronic specific heat contribution $\gamma(H) \cdot T$ in the mixed state. Thus, for an $\mathrm{Y}\left(\mathrm{Ni}_{0.8} \mathrm{Pt}_{0.2}\right){ }_{2} \mathrm{~B}_{2} \mathrm{C}$ single crystal $\gamma(H) \propto H$ has been found, while a square-root law was observed for a pure $\mathrm{YNi}_{2} \mathrm{~B}_{2} \mathrm{C}$ single crystal and for polycrystalline $\mathrm{LuNi}_{2} \mathrm{~B}_{2} \mathrm{C}$ [0]

$$
\gamma(H) / \gamma_{N} \propto \sqrt{H / H_{c 2}(0)},
$$

where $\gamma_{N}$ is the Sommerfeld constant in the normal state. Although the observed $\gamma(H) \propto \sqrt{H}$ law for $\mathrm{YNi}_{2} \mathrm{~B}_{2} \mathrm{C}$ and $\mathrm{LuNi}_{2} \mathrm{~B}_{2} \mathrm{C}$ was regarded initially as evidence for $d$-wave pairing [5.,6], the disorder related transition from a $\sqrt{H}$ to a linear-in- $H$ dependence was subsequently used to rule out $d$-wave superconductivity in non-magnetic borocarbides [4]. However, to the best of our knowledge, systematic investigations of this problem in a broader concentration range for $\mathrm{Y}\left(\mathrm{Ni}_{1-y} \mathrm{Pt}_{y}\right)_{2} \mathrm{~B}_{2} \mathrm{C}$ are lacking. Since isoelectronic substitutions in the $R \mathrm{C}$ charge reservoir are expected to produce much weaker disorder than those in the $T \mathrm{~B}$ network we studied also the closely related $\mathrm{Y}_{x} \mathrm{Lu}_{1-x} \mathrm{Ni}_{2} \mathrm{~B}_{2} \mathrm{C}$ system for the sake of comparison [7]. By changing both compositions, $x$ and $y$, deeper insight should be gained on how does the disorder affect the field dependence of the specific heat $c_{p}(T, H)$, the shape and the magnitude of $H_{c 2}(T)$, as well as the nature of the pairing state.

EXPERIMENTAL DETAILS. - Polycrystalline $\mathrm{Y}_{x} \mathrm{Lu}_{1-x} \mathrm{Ni}_{2} \mathrm{~B}_{2} \mathrm{C}$ with $x=0,0.25$, $0.5,0.75,1$ and $\mathrm{Y}\left(\mathrm{Ni}_{1-y} \mathrm{Pt}_{y}\right)_{2} \mathrm{~B}_{2} \mathrm{C}$ samples with $y=0,0.05,0.1,0.15,0.2,0.25,0.5$ and 0.75 were prepared by a standard arc melting technique. Powders of the elements were weighted in the stoichiometric compositions with a surplus of $10 \mathrm{wt} . \%$ boron to compensate losses of boron during arc melting. The powder was pressed to pellets which were melted in argon gas on a water-cooled copper plate in an arc furnace. To get homogeneous samples, they were turned over and melted again four times. After the melting procedure the solidified samples were homogenised at $1100{ }^{\circ} \mathrm{C}$ for ten days. The specific heat was measured in the range $4.2 \mathrm{~K} \leq T \leq 20 \mathrm{~K}$ and for magnetic fields $\mu_{0} H \leq 8 \mathrm{~T}$ using a quasi-adiabatic step heating technique. The heating pulses were generated by a strain gauge heater and $T$ was measured with a Au-Ge thin film resistor. The temperature dependence of the upper critical field $H_{c 2}(T)$ was determined by taking $T_{c}$ from the jump of $c_{p}$ in the particular field.

RESULTS AND DISCUSSION. - To illustrate typical specific heat behaviour, the $c_{p} / T$ vs $T^{2}$ data at $H=0$ of the $\mathrm{Y}_{x} \mathrm{Lu}_{1-x} \mathrm{Ni}_{2} \mathrm{~B}_{2} \mathrm{C}$ series and the corresponding curves for $\mu_{0} H \leq 8 \mathrm{~T}$ of the pure $\mathrm{Y}$ sample $(x=1)$ are shown in fig. 1. Measurements at $8 \mathrm{~T}$ were used to analyse the normal state specific heat $c_{p}=\gamma_{N} T+\beta_{D} T^{3}$, where $\gamma_{N}$ is the Sommerfeld constant and $\beta_{D} T^{3}$ is the Debye contribution. The $\gamma_{N}$ was determined by extrapolating the $c_{p} / T$ vs $T^{2}$ curves of the high field data in the normal state to $T \rightarrow 0$. In this way we obtained $\gamma_{N}=$ $20.4(x=0), 19.0(x=0.25), 18.3(x=0.5), 18.0(x=0.75)$ and $20.2 \mathrm{~mJ} / \mathrm{molK}^{2}(x=1)$ for our $\mathrm{Y}_{x} \mathrm{Lu}_{1-x} \mathrm{Ni}_{2} \mathrm{~B}_{2} \mathrm{C}$ series in good agreement with the data reported by several groups 88 14] and $\gamma_{N}=20.2(y=0), 20.2(y=0.05), 18.4(y=0.1), 16.4(y=0.15), 16.2(y=0.2), 16.9$ $(y=0.25), 15.3(y=0.5)$ and $15.0 \mathrm{~mJ} / \mathrm{molK}^{2}(y=0.75)$ for the $\mathrm{Y}\left(\mathrm{Ni}_{1-y} \mathrm{Pt}_{y}\right)_{2} \mathrm{~B}_{2} \mathrm{C}$ series. To determine $\gamma(H)$, the $c_{p} / T$ vs $T^{2}$ curves in the particular field have been extrapolated in the same way to $T \rightarrow 0$ from the data in the range $4.2 \mathrm{~K} \leq T \leq 7 \mathrm{~K}$ or up to the onset of the transition to the normal state (see fig. 2).

For all samples $\gamma(H)$ is a sublinear function of $H$. At first generalizing eq. (1), the data were analysed by the expression

$$
\gamma(H) / \gamma_{N}=\left[H / H_{c 2}(0)\right]^{1-\beta},
$$



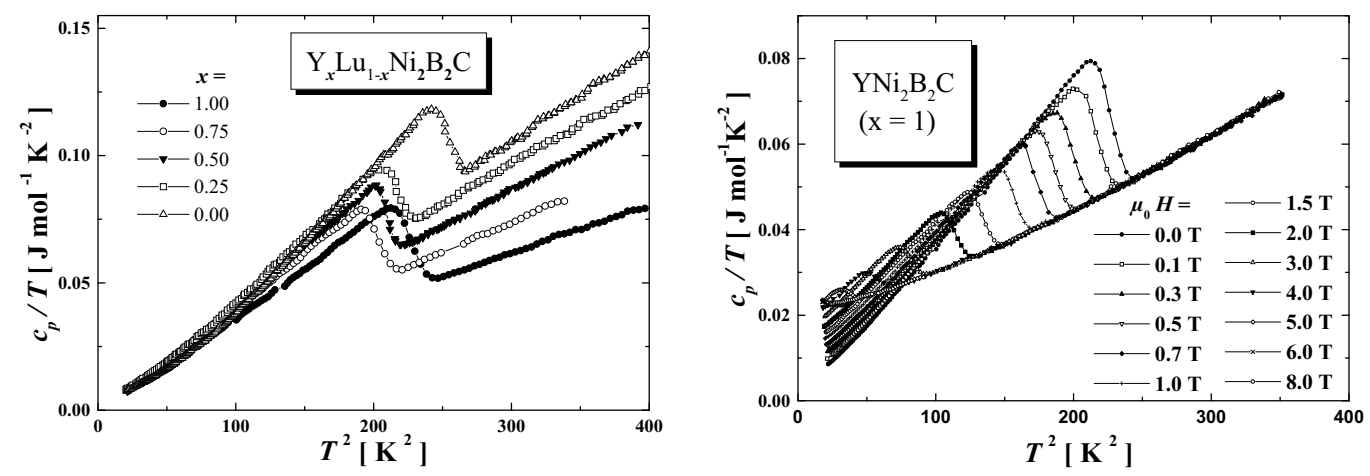

Fig. 1 - Zero magnetic field specific heat $c_{p}(T) / T$ vs $T^{2}$ of the $\mathrm{Y}_{x} \mathrm{Lu}_{1-x} \mathrm{Ni}_{2} \mathrm{~B}_{2} \mathrm{C}$ series (left panel) and specific heat $c_{p}(T, H) / T$ vs $T^{2}$ of $\mathrm{YNi}_{2} \mathrm{~B}_{2} \mathrm{C}$ for various magnetic fields (right).

where the fitting parameter $\beta$ measures the sublinearity (i.e. a negative curvature) of $\gamma(H)$ and $H_{c 2}(0)$ is the field where $\gamma(H)$ reaches $\gamma_{N}$. We obtained $\beta=0.66,0.42,0.42,0.41$ and 0.56 ongoing from $x=0$ to $x=1$ for $\mathrm{Y}_{x} \mathrm{Lu}_{1-x} \mathrm{Ni}_{2} \mathrm{~B}_{2} \mathrm{C}$ and $\beta=0.56,0.36,0.35,0.26,0.18$, $0.20,0.49$ and 0.60 ongoing from $y=0$ to $y=0.75$ for $\mathrm{Y}\left(\mathrm{Ni}_{1-y} \mathrm{Pt}_{y}\right)_{2} \mathrm{~B}_{2} \mathrm{C}$, with uncertainties of $\Delta \beta / \beta \leq 10 \%$ due to the small measured values $\gamma_{0}=\gamma(H=0)$ 15 and due to the procedure used to determine $\gamma(H)$, as mentioned above. The dependence of $\beta(x)$ is shown in the inset of fig. 2 and in fig. 1 (left panel). $\beta$ reaches the largest values for the bordering cases $x=0$ and 1 and becomes markedly smaller in between. We note that our curvatures for $\mathrm{LuNi}_{2} \mathrm{~B}_{2} \mathrm{C}$ and $\mathrm{YNi}_{2} \mathrm{~B}_{2} \mathrm{C}$ exceed slightly the value of $\beta=0.5$ suggested by eq. (11) and that reported in Refs. 44.5]. To the best of our knowledge the strong sublinearities for $\gamma(H)$, measured by the exponent $\beta$, of the borocarbides under consideration are the largest reported so far for any superconductor. The $\beta(y)$ behaviour is depicted in the inset of fig. 2 and in fig. (1) (right panel). Large exponents $\beta \approx 0.5$ are observed for $y=0$ and $y=0.5$, whereas $\beta$ is significantly reduced for Pt-concentrations $y$ inside this range and has a finite minimum value $\beta=0.18$ at $y=0.2$. This minimum value is at variance with the linear law for an $\mathrm{Y}\left(\mathrm{Ni}_{0.8} \mathrm{Pt}_{0.2}\right)_{2} \mathrm{~B}_{2} \mathrm{C}$ single crystal reported in ref. [ [ 1 . We attribute that observation to a stronger disorder compared with our samples. Larger Pt-concentrations reveal even more pronounced $\beta$ values, e.g. $y=0.75$; $\beta=0.6$.

The observed $\gamma(H) \propto H^{1-\beta}$-law with $\beta \approx 0.5$ raises the question whether an unconventional pairing mechanism is responsible for this peculiarity since according to Ref. [16] $\gamma(H) \propto \sqrt{H}$ is a signature for a nodal order parameter with $d$-wave symmetry (a somewhat larger value $\beta=0.59$ has been found in Ref. [17]) while $\gamma(H) \propto H$ describes an isotropic $s$-wave order parameter. According to Refs. 18, 19 Volovik's clean limit $d$-wave approach can be generalized to describe also strong impurity scattering. Then at low magnetic fields $H \ll H_{c 2}(0)$ the specific heat coefficient $\gamma(H)$ follows a $H \ln H$ dependence:

$$
\gamma(H)=\gamma_{0}+\gamma_{N} D\left(\frac{H}{H_{c 2}(0)}\right) \ln \left[\frac{\pi}{2 a^{2}}\left(\frac{H_{c 2}(0)}{H}\right)\right],
$$

where $a$ and $D$ are constants. Such a behaviour was observed for various disordered high- $T_{c}$ cuprates and considered as evidence for $d$-wave superconductivity in the unitary scattering limit 20,21]. At the same time its applicability to non-magnetic borocarbides under consideration was disclaimed [20]. However, some of our data can be described equally well by eq. (3) 

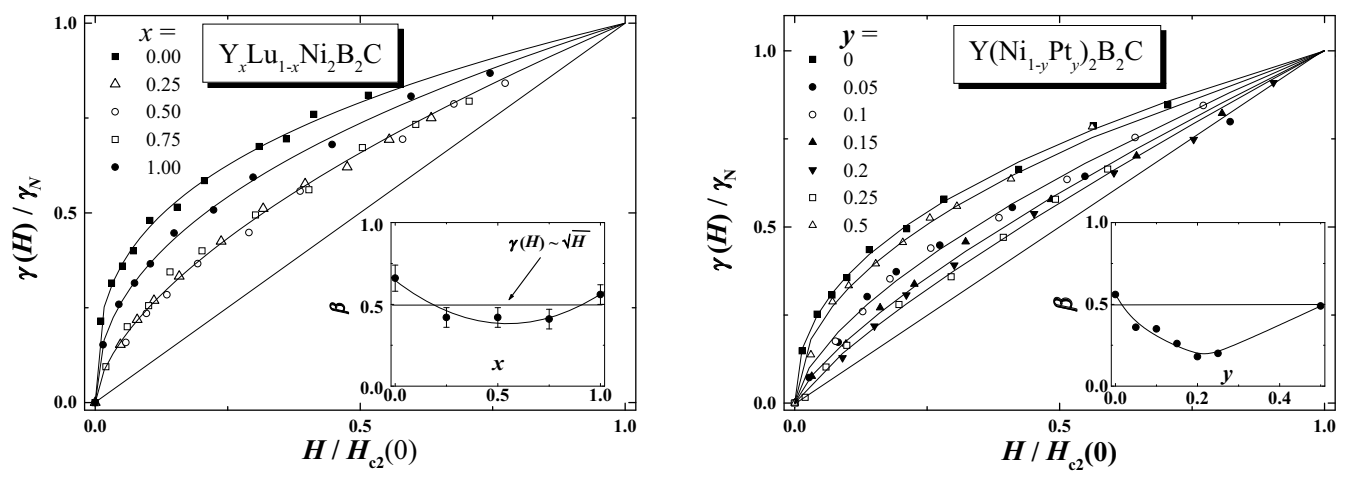

Fig. 2 - Magnetic field dependence of the specific heat contribution $\gamma(H)$ of the vortex core electrons in the mixed state $\left(H \leq H_{c 2}\right)$ normalised by $\gamma_{N}$ with $H_{c 2}(0)$ as the upper critical field (see fig. 1 ) for $\mathrm{Y}_{x} \mathrm{Lu}_{1-x} \mathrm{Ni}_{2} \mathrm{~B}_{2} \mathrm{C}$ (left panel) and $\mathrm{Y}\left(\mathrm{Ni}_{1-y} \mathrm{Pt}_{y}\right)_{2} \mathrm{~B}_{2} \mathrm{C}$ (right panel). The lines are fits according to eq. (2) and the straight reference line corresponds to the usual linear-in- $H s$-wave dirty limit behaviour. The insets show the curvature parameters $\beta(x)$ and $\beta(y)$ as defined in eq. (2).

for $H / H_{c 2}(0) \leq 0.3$ as well as by eq. (2) using intermediate values for $\beta$ (0.2 to 0.35$)$. This is shown in fig. 3: obviously, the $H \ln H$ behaviour is not very distinct from the power law at low fields $\mu_{0} H \leq 1.5 \mathrm{~T}$. At higher fields the $H \ln H$ dependence may deviate since it was derived for low fields only [18]). For $y=0.25$ and 0.5 we observed residual values $\gamma_{0}=3.4$ and 3.3 $\mathrm{mJ} / \mathrm{molK}^{2}$, respectively, which are substracted in fig. 2[15]. The existence of a non-negligible $\gamma_{0}$ is a feature predicted for a $d$-wave order parameter in the unitary limit [22]. Hence, $d$-wave pairing cannot be ruled out in non-magnetic borocarbides by considering $\gamma(H)$ data only. While the deviation from the linearity of $\gamma(H)$ is frequently ascribed to a shrinking of the

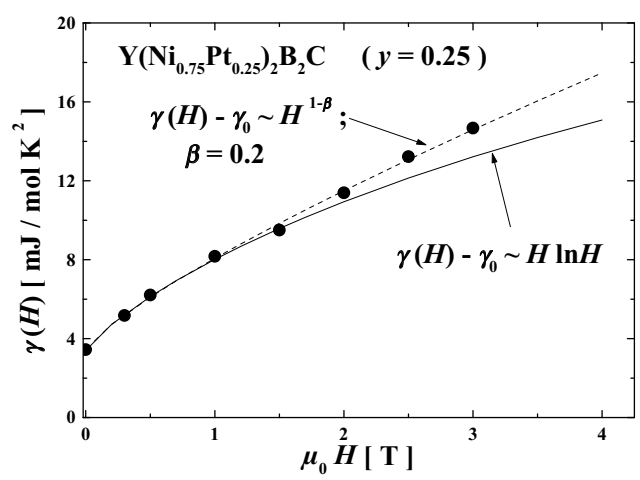

Fig. 3 - Magnetic field dependence of $\gamma(H)$ for $\mathrm{Y}\left(\mathrm{Ni}_{0.75} \mathrm{Pt}_{0.25}\right)_{2} \mathrm{~B}_{2} \mathrm{C}$. The solid line is a fit according to eq. (3). The dashed line is a fit according to eq. (2) with $\beta=0.20$.

vortex cores with magnetic field and to vortex core interactions [4, 23, 24], recent investigations support the assumption of delocalized quasiparticle states around the vortex core to be responsible for this feature, similar as in $d$-wave superconductors 25]. However, there are several conventional, but anisotropic $s$-wave, superconductors which also exhibit deviations from the $\gamma(H) \propto H$-law in the clean limit, e.g. $\mathrm{V}_{3} \mathrm{Si}$ [26], $\mathrm{NbSe}_{2}$ [⿴囗十 $(\beta=0.33)$, and $\mathrm{CeRu}_{2}$ [17,27]. 
Remarkably, a sublinear $\gamma(H)$ behaviour has been reported also for the novel "medium- $T_{c}$ " superconductor $\mathrm{MgB}_{2}$ [28]. In this general context recent ultrahigh-resolution photoemission spectroscopy measurements suggest that a highly anisotropic gap might be responsible for the mentioned above peculiarities in clean Ni borocarbides 29]. By introducing disorder $(y=0.2)$ a complete isotropization of the gap was observed. Calculations of the density of states (DOS) at the Fermi level, $N(0)$, in the mixed state with interacting vortices by Ichioka et al. [17] revealed a $H^{0.67}(\beta=0.33)$ dependence of $\gamma(H)$ for anisotropic $s$-wave superconductors.

In this context it is noteworthy that our low-temperature $c_{p}$ data on $\mathrm{YNi}_{2} \mathrm{~B}_{2} \mathrm{C}(x=1)$ point to a possible exponential behaviour of $c_{e s}(T)$ below $1 / 6 T_{c}$ with $\Delta(0) / k_{B} T_{c} \approx 0.5$. This supports an effective multiband $s$-wave picture like that proposed by Shulga et al. 30]. Noteworthy, a similar value for $\Delta(0)$ was observed in microwave measurements [31].

Like $\gamma(H)$, the upper critical field $H_{c 2}(T)$ can be described also by a simple scaling law [32]

$$
H_{c 2}(T)=H_{c 2}^{*}\left(1-T / T_{c}\right)^{1+\alpha}, \quad \text { valid for } \quad 0.3 \leq T / T_{c} \text {. }
$$

Our values of the upper critical field $H_{c 2}(0) \approx 0.9 H_{c 2}^{*}$ are reduced due to R-site substitution. A similar behaviour was found for the pronounced positive curvature of $H_{c 2}(T)$ near $T_{c}$, which is measured by the exponent $\alpha$ in eq. (4), in contrast to the opposite statement of a nearly constant curvature [8]. The unusual positive curvature of $H_{c 2}(T)$ near $T_{c}$ observed here can be explained for superconductors in the clean limit by a significant dispersion of the Fermi velocities using e.g. an effective two-band model [30. $T_{c}$ and $\gamma_{N}$ are reduced to a smaller extent, which has been ascribed to a slight reduction of the electron-phonon coupling constant $\lambda$ at intermediate $x$ [33, 34]. For $T_{c}$ a dip near $x=0.7$ is observed, in accordance with refs. [8, 32 $\left(T_{c} \approx 14.6 \mathrm{~K}\right.$ at $\left.x=0.75\right)$. The dirty limit region is not reached (which would be represented by vanishing $\alpha$ and increasing $H_{c 2}(0)$ with increasing disorder [9, 10]

In the case of Pt-substitutions in the investigated range $0<y<0.75$, the values of $T_{c}, \beta$ and $H_{c 2}(0)$ are reduced, too. As for R-substitutions, those superconducting properties exhibit minima at intermediate composition while the Sommerfeld constant $\gamma_{N}$ and the curvature parameter $\alpha$ of $H_{c 2}(T)$ depend monotonically on $y$. For $y<0.2$ a strong decrease of $\alpha$ with increasing $y$ is observed, but for $y>0.2$ an increase of $\alpha$ does not occure (see fig. (4)). This behaviour of $H_{c 2}$ suggests that the quasi-dirty limit has been reached at about $y \approx 0.2$ since $H_{c 2}(0)$ increases above $y \approx 0.2$, while the curvature of $H_{c 2}$ measured by $\alpha$ remains strongly reduced. The results obtained for $\mathrm{Y}\left(\mathrm{Ni}_{1-y} \mathrm{Pt}_{y}\right)_{2} \mathrm{~B}_{2} \mathrm{C}$ show that the deviations from the linearity of $\gamma(H)$ measured by $\beta$ are not correlated with the field exponent $\alpha$. While $\alpha$ almost vanishes, $\beta$ does increase for $y>0.2$. Thus, here the behaviour of the specific heat in the vortex state even in the quasi-dirty limit remains rather complex. In this context a similar behaviour for $(\mathrm{Nb}, \mathrm{Ti})$ alloys which are apparently strongly disordered superconductors is noteworthy: on one hand, a strong sublinear $\gamma(H) \propto H^{0.5}$-dependence is observed, and on the other hand, a standard parabolic Werthammer-Helfand-Hohenberg temperature dependence of $H_{c 2}(T)$ occures, i.e. a negative curvature near $T_{c}$ is present [35].

To summarize, we have shown that the $\gamma(H)$ curves of the pure specimens $(x=0 ; 1)$ of $\mathrm{Y}_{x} \mathrm{Lu}_{1-x} \mathrm{Ni}_{2} \mathrm{~B}_{2} \mathrm{C}$ exhibit the strongest sublinear behaviour reported for superconductors. Weak disorder effects caused by isoelectronic substitutions of Lu by $\mathrm{Y}$ yield a reduction of the $\gamma(H)$-non-linearity without reaching the standard linear behaviour. Similar moderate suppressions of characteristic features which are typical for the quasi-clean limit have been found for the upper critical field $H_{c 2}(0)$, the curvature exponent $\alpha, \gamma_{N}$ and $T_{c}$. Stronger disorder effects are caused by isoelectronic substitutions of $\mathrm{Ni}$ by $\mathrm{Pt}$. From the behaviour of $H_{c 2}(T)$ it is deduced that a transition from clean to quasi-dirty limit occures caused by 

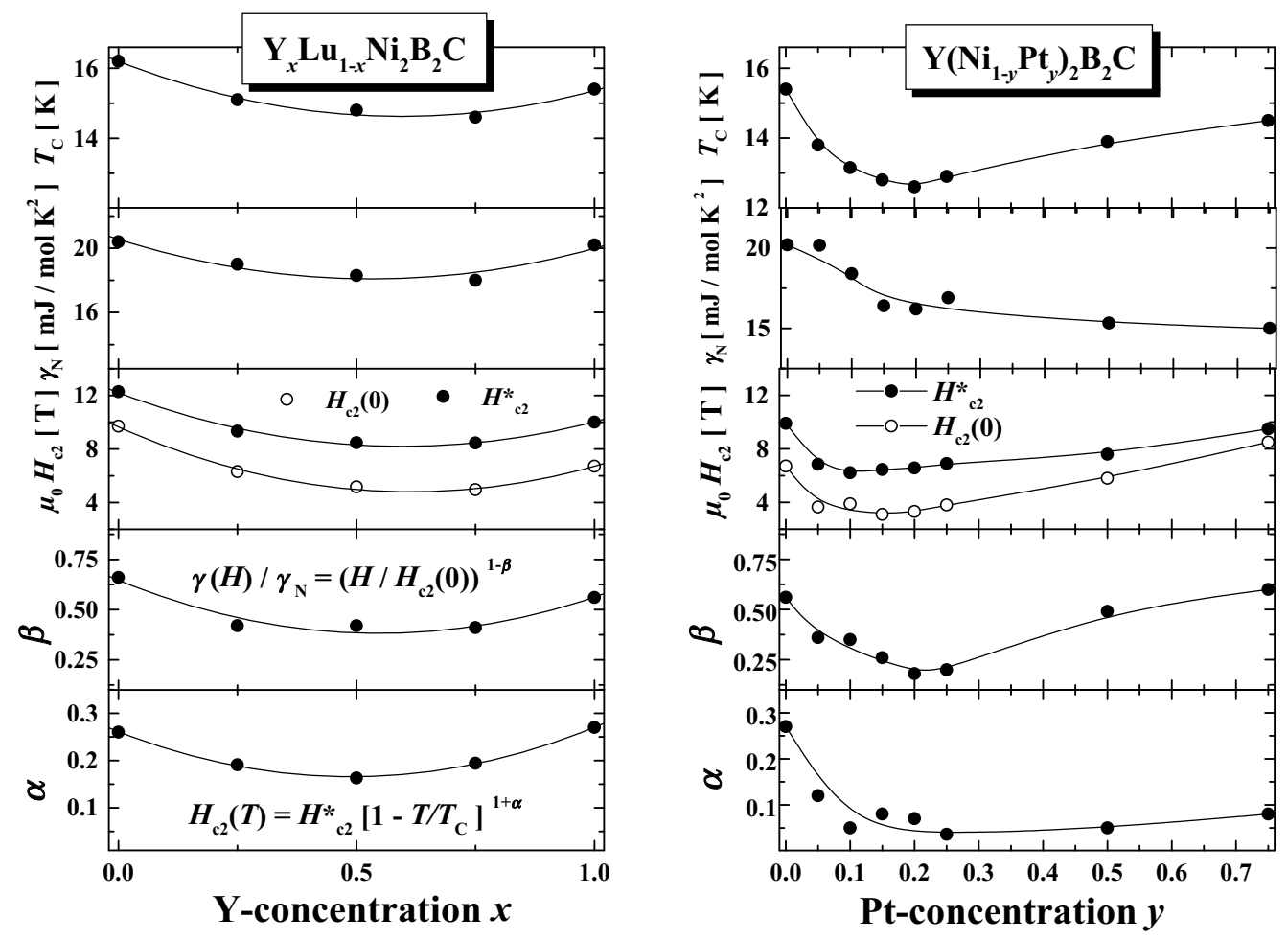

Fig. 4 - Composition dependence of the transition temperature $T_{c}$ derived from the onset of the jump of $c_{p}(T)$ (upper panels), the Sommerfeld constant $\gamma_{N}$ (second panels), the upper bound for the upper critical field $H_{c 2}^{*}$ according to eq. (4) and $H_{c 2}(0)$ according to eq. (2) (third panels, see text for more details), the specific heat curvature exponent $\beta$ of $\gamma(H)$ according to eq. (4) (fourth panels), and the curvature exponent $\alpha$ of the upper critical field $H_{c 2}$ according to eq. (何) (lower panels) determined for $\mathrm{Y}_{x} \mathrm{Lu}_{1-x} \mathrm{Ni}_{2} \mathrm{~B}_{2} \mathrm{C}$ (left) and for $\mathrm{Y}\left(\mathrm{Ni}_{1-y} \mathrm{Pt}_{y}\right)_{2} \mathrm{~B}_{2} \mathrm{C}$ (right). The lines are guides to the eye.

isoelectronical substitutions at the $T$-site. The quasi-dirty limit is deduced from the nearly vanishing curvature of $H_{c 2}(T)$ for $y \geq 0.2$. At the same time there the sublinearity of $\gamma(H)$ remains and does even increase. Hence, a simple monotonical relationship between $\alpha$ and $\beta$, as one might expect by considering the results on $\mathrm{Y}_{x} \mathrm{Lu}_{1-x} \mathrm{Ni}_{2} \mathrm{~B}_{2} \mathrm{C}$ only, does not hold in the quasi-dirty limit. In the case of intermediate deviations from the linearity of $\gamma(H)$ $(\beta=0.2-0.35)$ our results on specific heat at low magnetic fields are discussed in the context of a dirty $d$-wave model on the one hand and within the framework of the conventional $s$-wave picture in the quasi-clean limit on the other hand. At low fields the $H \ln H$ dependence of $\gamma(H)$ predicted for $d$-wave pairing in the dirty (unitary) limit is not very distinct from the $H^{1-\beta}$ behaviour which favoures $s$-wave superconductivity in the quasi-clean limit. Thus, results on $\gamma(H)$ only are insufficient to rule out unconventional pairing in borocarbide superconductors. But from the few available $c_{p}$ data points at low-temperature and $H=0$ a small gap of $\Delta(0) / k_{B} T_{c} \approx 0.5$ might be derived for a clean $\mathrm{YNi}_{2} \mathrm{~B}_{2} \mathrm{C}$ sample. This reveals additional support for an anisotropic $s$-wave pairing state in non-magnetic borocarbides. 
This work has been supported by the SFB 463 and the DFG. We acknowledge discussions with S. Shulga, H. Rosner, H. Michor, M. Nohara, K. Maki, H. Takagi, and D.G. Naugle as well as R. Botha for a critical reading of the manuscript.

\section{REFERENCES}

[1] Nagarajan R. et al., Phys. Rev. Lett., 72 (1994) 274.

[2] Cava R. et al., Letters to Nature, 367 (1994) 146.

[3] Drechsler S.-L. et al., Physica C, 317-318 (1999) 117.

[4] Nohara M., Isshiki M., Sakai F. and Takagi H., J. Phys. Soc. Jpn., 68 (1999) 1078.

[5] Nohara M., Isshiki M., Takagi H. and Cava R., J. Phys. Soc. Jpn., 66 (1997) 1888.

[6] Wang G. and Maki K., Phys. Rev. B, 58 (1998) 6493.

[7] Preliminary results for that particular case have been published in: LipP D. et al., Rare Earth Transition Metal Borocarbides (Nitrides): Superconducting, Magnetic and Normal State Properties, edited by K.-H. MÜller AND V. NAROzhnyI, Vol. 14 (Kluwer Academic Publishers, Dordrecht) 2001, p. 89; corresponding preprint, cond-mat/0010066 (2000).

[8] Manalo S. et al., Phys. Rev. B, 63 (2001) 104508.

[9] Fuchs G. et al., Rare Earth Transition Metal Borocarbides (Nitrides): Superconducting, Magnetic and Normal State Properties, edited by K.-H. Müller And V. NArozhnyi, Vol. 14 (Kluwer Academic Publishers, Dordrecht) 2001, p. 243.

[10] Drechsler S.-L. et al., Physica C, 341-348 (2000) 749.

[11] Michor H., Holubar T., Dusek C. and Hilscher G., Phys. Rev. B, 54 (1996) 9408.

[12] Moshovich R. et al., Physica C, 227 (1994) 381.

[13] Hilscher G. and Michor H., Studies of High Temperature Superconductors, edited by A. V. NARliKar, Vol. 28 (Nova Science Publishers, New York) 1999, p. 241.

[14] Carter S. et al., Phys. Rev. B, 50 (1994) 4216.

[15] For all our other samples small residual values $\gamma_{0} \leq 1.5 \mathrm{~mJ} / \mathrm{molK}^{2}$ are observed. These values are substracted in fig. 2, too.

[16] VoloviK G.E., JETP Lett., 58 (1993) 469.

[17] Ichioka M., Hasegawa A. and Machida K., Phys. Rev. B, 59 (1999) 184.

[18] Kübert C. and Hirschfeld P.J., Solid State Commun., 105 (1998) 459.

[19] Barash Y.S., Svidzinskit A.A. and Mineev V.P., JETP Lett., 65 (1997) 638.

[20] Nohara M. et al., J. Phys. Soc. Jpn., 69 (2000) 1602.

[21] Nohara M. et al., Physica C, 341-348 (2000) 2177.

[22] Preosti G. et al., Phys. Rev. B, 50 (1994) 1259.

[23] Sonier J.E. et al., Phys. Rev. Lett., 82 (1999) 4914.

[24] Ichioka M. et al., Phys. Rev. B, 59 (1999) 8902.

[25] Izawa K. et al., Phys. Rev. Lett., 86 (2001) 1327.

[26] Ramirez A.P., Phys. Lett., 211 (1996) 59.

[27] Hedo M. et al., J. Phys. Soc. Jpn., 67 (1998) 33; 67 (1998) 272.

[28] Wang Y., Plackowski T. and Junod A., preprint, cond-mat/0103181 (2001).

[29] Yokoya T. et al., Phys. Rev. Lett., 85 (2000) 4952.

[30] Shulga S.V. et al., Phys. Rev. Lett., 80 (1998) 1730.

[31] Jacobs T. et al., Phys. Rev. B, 52 (1995) R7022.

[32] Freudenberger J. et al., Physica C, 306 (1998) 1.

[33] Drechsler S.-L. et al., J. Low Temp. Phys., 117 (1999) 1617.

[34] Freudenberger J. et al., J. Low Temp. Phys., 117 (1999) 1623.

[35] LiPP D. , PH-Thesis, (2001) TU Dresden, unpublished. 